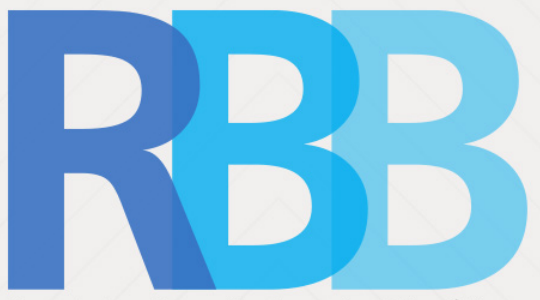

Revista Brasileira de Bioética

Mário de Seixas Rocha

Escola Bahiana de Medicina e Saúde

Pública, Salvador, BA, Brasil

entouragemobile@me.com

Simone Azevedo Rocha

UNIRB, Salvador, BA, Brasil

simoneadvazevedo@yahoo.com.br

\section{Resolução de Conflitos Bioéticos no Cenário Hospitalar Brasileiro: Uma Revisão Sistemática da Literatura}

\author{
Bioethical Conflict Resolution in the Brazilian Hospital Setting: A \\ Systematic Review of Literature
}

Resumo: Os conflitos bioéticos constituem uma parte dilemática da atividade assistencial contemporânea. Tomar decisões acertadas frente a um problema moral é de extrema importância no cenário hospitalar. A partir de uma revisão sistemática da literatura, objetivou-se avaliar as estratégias para resolução dos conflitos éticos, verificando-se se houve a participação de comitês de bioética hospitalar e utilização de metodologias de auxilio de tomada de decisão em hospitais brasileiros. O estudo revela que há ainda um número inexpressivo de comités de aconselhamento bioético nas instituições de saúde do país, demonstrando a necessidade de estimular-se a sua criação como mecanismo de auxílio aos profissionais de saúde na tomada de decisão em casos clínicos mais complexos.

Palavras-chave: Bioética, ética clínica, ética médica, tomada de decisão.

Abstract: Bioethical conflicts constitute a dilemmatic part of contemporary care activity. Making sound decisions in the face of a moral problem is of the utmost importance. From a systematic review of the literature, the objective was to evaluate strategies for conflict resolution. Evaluating the incorporation of hospital ethics committees and decision-making methodologies in Brazilian hospitals. This study reveals there is still an inexpressive number of bioethics committees in the country. This data, in turn, demonstrates the necessity to incentive the creation of bioethics committees as a mechanism to assist medical professionals in their making of decisions for complex clinical cases.

Keywords: Bioethics, clinical ethics, medical ethics, decision-making. 


\section{Introdução}

A tomada de decisão pode ser extremamente difícil para o profissional da área de saúde, em especial quando envolve conflitos bioéticos entre pacientes, familiares, instituições de saúde e equipe médica assistencial. Muitas destas tomadas de decisão abordam questões relativas ao início da vida, final da vida, alocação de recursos e realização de procedimentos terapêuticos (16).

O instrumental necessário para resolução dos dilemas biomédicos pode ser extremamente complexo, tendo em vista a necessidade de envolvimento de diferentes áreas do conhecimento, como ciências da saúde, humanas e aplicadas (2).

O processo decisório envolve várias etapas como: 1) a decisão de decidir; 2) a definição do que se vai se decidir; 3) a formulação de alternativas; 4) a escolha da alternativa mais adequada (3).

A tomada de decisão deve ser a mais correta para o paciente, não implicando apenas na adequação técnica e científica, mas aquela que ofereça o melhor benefício para o paciente dentro de suas convicções e seus valores (24).

Dilemas, que requerem decisões morais complexas, necessitam de mecanismos formais de enfrentamento que abordem e forneçam respostas adequadas para os pacientes. Assim, em face dessas complexidades, os diferentes atores envolvidos no processo necessitam de uma instância de aconselhamento para que a melhor solução seja tomada diante do conflito ético criado (12).

Um mecanismo sugerido para mediar conflitos entre os profissionais de saúde, pacientes e familiares são os comitês de bioética hospitalar, constituídos por equipes interdisciplinares que têm por objetivo ensinar, pesquisar, prestar consultorias e sugerir normas institucionais em assuntos éticos (12).

Esses comitês são compostos frequentemente por médicos de diferentes especialidades, enfermeiros, assistentes sociais, psicólogos, representantes da comunidade e advogados, que fornecem aconselhamento ético e alternativas moralmente aceitáveis (6). Os comitês têm se tornado muito comuns em países ocidentais, particularmente Estados Unidos (22), Canadá, tendo por fim o atendimento de acreditação hospitalar internacional (20).

O processo de deliberação moral dos dilemas nos comitês de ética hospitalar é facilitado pela utilização de métodos de auxílio a tomada de decisão elaborados por diferentes autores (15). 
Esses métodos configuram-se como estruturas formais de tomada de decisão que ajudam as pessoas na identificação de todas as partes interessada. Permite-se, assim, discussões sistemáticas e completas sem que nada relevante seja esquecido. Eles podem ajudar a minimizar o desconforto inevitável ao longo de discussões de dilemas éticos difíceis; assegurando, dessa forma, um bom processo de tomada de decisão de forma que todos os envolvidos possam compreender o processo de deliberação, mesmo quando não concordem integralmente com a decisão tomada (1).

Como já informado, esses métodos permitem a análise dos dilemas e facilitam o processo, a partir de um exame da história clínica em questão, da identificação dos problemas, da análise dos fatos, da identificação dos valores em conflito, da elucidação do conflito fundamental, com isso delibera-se, expressa-se a tomada de decisão e identifica-se se a decisão é antijurídica (6).

Em um cenário nacional, onde as estratégias de resolução de dilemas éticos são pouco conhecidas, este artigo tem como objetivo: 1) identificar na literatura nacional evidências que sugiram a prática de resolução de dilemas éticos que utilizaram ou sugeriram análise pelos comitês de ética hospitalar; 2) verificar se houve a utilização de metodologias de auxílio para tomada de decisão; 3 ) apresentar reflexões sobre o processo decisório nas situações identificadas.

\section{Metodologia}

Foi realizada uma pesquisa sistemática da literatura na base de dados LILACS - Literatura Latino-americana e do Caribe em Ciências da Saúde, de 2001 a 2016. A estratégia de busca incluiu"bioética" ou "bioethics", "ética clínica" ou "clinical ethics", "ética médica" ou "ethics medical", "tomada de decisão" ou "decision-making". As palavras chave foram buscadas nos descritores de ciências e saúde (DeCS) e MeSH Pubmed. Os descritores foram utilizados de forma combinada, sendo eles: "bioética" AND "tomada de decisão", "bioethics" AND "decision-making", "ética clínica" AND "tomada de decisão", "clinical ethics", AND "decision-making", "ética médica" AND "tomada de decisão", "ethics medical" AND "decision-making".

Os critérios de inclusão foram: (I) artigos científicos disponíveis que discorressem sobre resolução de conflitos no âmbito de hospitais brasileiros; (II) intervalo de busca no período de 2001 a 2016; (III) sendo publicado, como um documento completo, no idioma inglês ou português e foram excluídos, estudos que não atendiam aos critérios estabelecidos, os países de afiliação diferente do Brasil, bem como aqueles que apesar de apresentarem os descritores selecionados não abordavam a temática proposta, além de teses, dissertações e monografias sobre o assunto. 
Os artigos foram selecionados, inicialmente, pelos títulos e resumos, para estabelecer a relevância, e, em sequência, foi feita a leitura completo do documento, para verificar se atendia aos critérios de inclusão. Todas as publicações foram analisadas integralmente por dois pesquisadores. Quaisquer discrepâncias foram resolvidas através da discussão.

Os dados foram extraídos a partir de todos os estudos incluídos, utilizando uma forma estruturada: (I) Título; (II) Ano de publicação; (III) Tipo do estudo; (IV) Presença no texto de abordagem dirigida à questões de interesse; (V) Resultados.

\section{Resultados}

A pesquisa bibliográfica resultou em 97 artigos na base de dados LILACS, tendo sido excluídos 6 artigos por serem duplicados, 57 foram selecionados após leitura do título e do resumo, foram excluídos 34por não descreverem a questão em estudo. Portanto, 23artigos foram avaliados após a leitura do texto completo. Após a leitura, foram eliminados 10 artigospor fugirem ao escopo da revisão. Assim, 15 artigos forma incluídos na revisão sistemática. A Figura 1 resume o fluxograma de artigos incluídos.

Figura 1: Revisão sistemática de artigos.

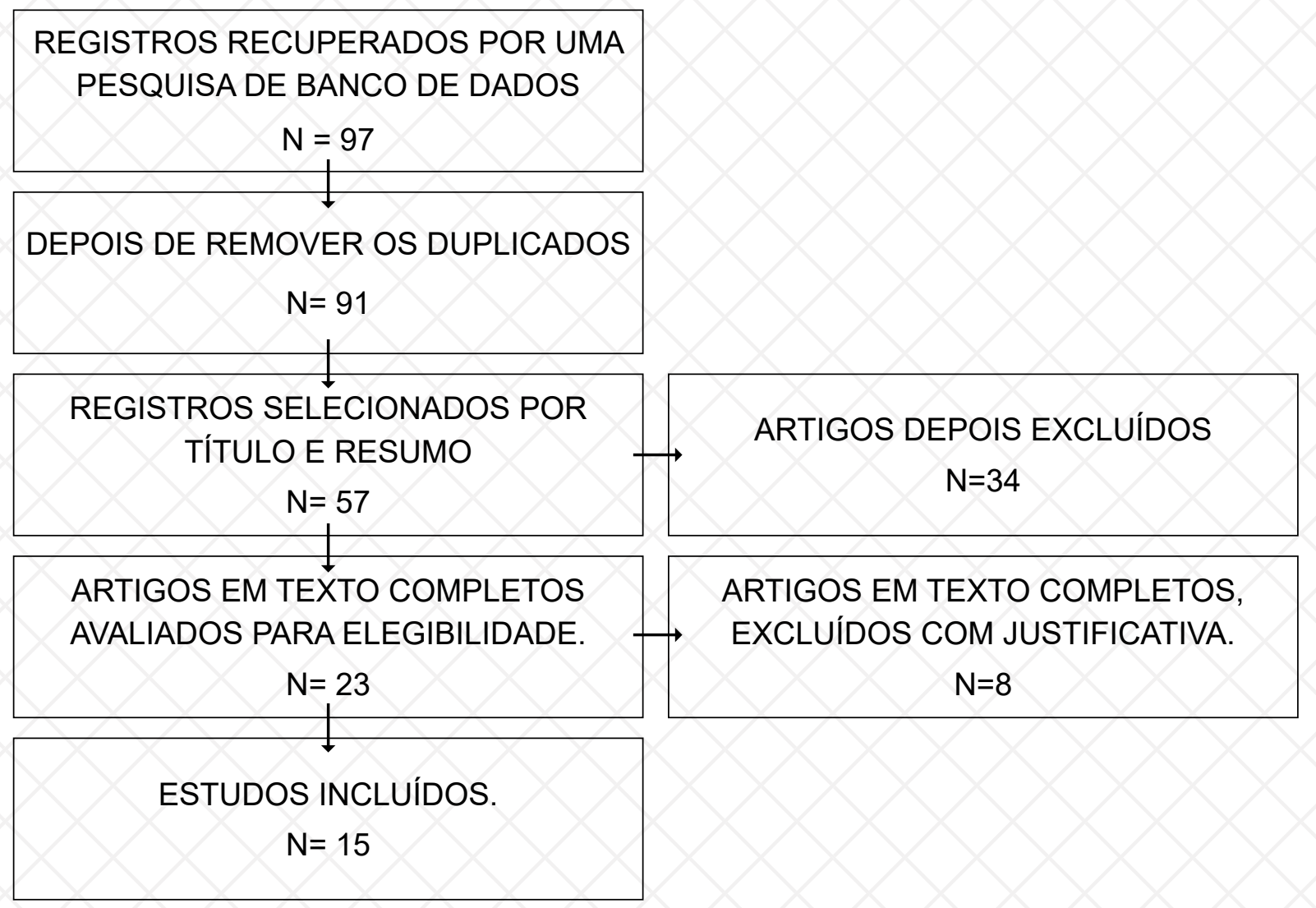


A Tabela 1 apresenta as características dos estudos selecionados, ordenados por ano de publicação de janeiro de 2001 a dezembro de 2016 (15 anos).

Tabela 1: Características dos artigos selecionados.

\begin{tabular}{|c|c|c|c|}
\hline TÍTULO & LUGAR & ESTUDO & CENARIO \\
\hline $\begin{array}{l}\text { Fortes et } \\
\text { al/2001 }\end{array}$ & São Paulo/SP & Transversal 1 & Estudantes universitários \\
\hline $\begin{array}{l}\text { Carvalho et } \\
\text { al/2001 }\end{array}$ & $\begin{array}{l}\text { Porto Alegre/ } \\
\text { RS }\end{array}$ & Retrospectivo & $\begin{array}{l}\text { Pacientes de UTI aspectos } \\
\text { sobre o modo de morrer }\end{array}$ \\
\hline $\begin{array}{l}\text { França et } \\
\text { al/2008 }\end{array}$ & $\begin{array}{l}\text { Campina } \\
\text { Grande/PB }\end{array}$ & $\begin{array}{l}\text { Revisão } \\
\text { Sistemática }\end{array}$ & $\begin{array}{l}\text { Hemotransfusão em } \\
\text { Testemunhas de Jeová }\end{array}$ \\
\hline $\begin{array}{l}\text { Ribeiro e } \\
\text { Rego/2008 }\end{array}$ & Niterói/RJ & $\begin{array}{l}\text { Revisão } \\
\text { Narrativa }\end{array}$ & UTIs Neonatais \\
\hline $\begin{array}{l}\text { Wittman-Viera } \\
\text { et al/2008 }\end{array}$ & $\begin{array}{l}\text { Porto Alegre/ } \\
\text { RS }\end{array}$ & Transversal 1 & $\begin{array}{l}\text { Unidade de cuidados } \\
\text { paliativos }\end{array}$ \\
\hline $\begin{array}{l}\text { Fortes et } \\
\text { al/2012 }\end{array}$ & São Paulo/SP & $\begin{array}{l}\text { Pesquisa } \\
\text { qualitativa }\end{array}$ & Emergências médicas \\
\hline $\begin{array}{l}\text { Freitas et } \\
\text { al/2013 }\end{array}$ & $\begin{array}{l}\text { Rio de } \\
\text { Janeiro/RJ }\end{array}$ & $\begin{array}{l}\text { Revisão } \\
\text { Sistemática }\end{array}$ & $\begin{array}{l}\text { Idosos na atenção à } \\
\text { saúde em UTIs }\end{array}$ \\
\hline $\begin{array}{l}\text { Zoboli et } \\
\text { al/2013 }\end{array}$ & São Paulo/SP & $\begin{array}{l}\text { Revisão } \\
\text { Narrativa }\end{array}$ & $\begin{array}{l}\text { Procedimentos de tomada } \\
\text { de decisão }\end{array}$ \\
\hline $\begin{array}{l}\text { Siqueira- } \\
\text { Batista et } \\
\text { al/2014 }\end{array}$ & Viçosa/MG & $\begin{array}{l}\text { Revisão } \\
\text { Sistemática }\end{array}$ & $\begin{array}{l}\text { Abordagem computacional } \\
\text { na tomada de decisão }\end{array}$ \\
\hline $\begin{array}{l}\text { Bezerra et al/ } \\
2014\end{array}$ & Recife/PE & $\begin{array}{l}\text { Revisão } \\
\text { Sistemática }\end{array}$ & $\begin{array}{l}\text { Decisão na Prematuridade } \\
\text { extrema }\end{array}$ \\
\hline $\begin{array}{l}\text { Gomes et } \\
\text { al/2014 }\end{array}$ & $\begin{array}{l}\text { Belo } \\
\text { Horizonte/MG }\end{array}$ & $\begin{array}{l}\text { Revisão } \\
\text { Narrativa }\end{array}$ & $\begin{array}{l}\text { Limitação do esforço } \\
\text { terapêutico na lesão } \\
\text { encefálica grave }\end{array}$ \\
\hline $\begin{array}{l}\text { Kipper et } \\
\text { al/2015 }\end{array}$ & $\begin{array}{l}\text { Porto Alegre/ } \\
\text { RS }\end{array}$ & $\begin{array}{l}\text { Relato de } \\
\text { caso }\end{array}$ & $\begin{array}{l}\text { Limites do poder familiar } \\
\text { nas decisões sobre saúde } \\
\text { de seus filhos }\end{array}$ \\
\hline $\begin{array}{l}\text { Lima et } \\
\text { al/2015 }\end{array}$ & $\begin{array}{l}\text { Rio de } \\
\text { Janeiro/RJ }\end{array}$ & $\begin{array}{l}\text { Rio de } \\
\text { Janeiro/ RJ }\end{array}$ & Rio de Janeiro/ RJ \\
\hline $\begin{array}{l}\text { Pastura et } \\
\text { al/2016 }\end{array}$ & $\begin{array}{l}\text { Rio de } \\
\text { Janeiro/RJ }\end{array}$ & $\begin{array}{l}\text { Revisão } \\
\text { Narrativa }\end{array}$ & $\begin{array}{l}\text { Procedimentos de tomada } \\
\text { de decisão }\end{array}$ \\
\hline $\begin{array}{l}\text { Motta et } \\
\text { al/2016 }\end{array}$ & $\begin{array}{l}\text { Teresópolis/ } \\
\text { RJ }\end{array}$ & $\begin{array}{l}\text { Revisão } \\
\text { Sistemática }\end{array}$ & $\begin{array}{l}\text { Procedimentos de tomada } \\
\text { de decisão }\end{array}$ \\
\hline
\end{tabular}


É importante assinalar que todos os quinze artigos foram publicados em língua portuguesa $(100 \%)$. Em relação ao ano de publicação, verificou-se que dois $(13 \%)$ foram publicados em 2001, três (20\%) em 2008, um (7\%) em 2012, dois (13\%) em 2013, três (20\%) em 2014, dois (13\%) em 2015 e dois (13\%) em 2016.

Quanto a síntese de publicação encontradas na base de dados foi possível agrupá-las conforme o tema abordado, início da vida em quatro (27\%), final da vida em três $(20 \%)$, alocação de recursos em três $(20 \%)$, realização procedimentos terapêuticos em um (6\%) e estratégias de tomada de decisão em quatro $(27 \%)$.

\section{Discussão}

A discussão é apresentada de acordo com a temática dos artigos, construção sintética dos cenários em que foram realizados os estudos, finalizando com obtenção de evidencia sobre comitês de bioética hospitalar e utilização de metodologias para resolução dos conflitos bioéticos.

\section{Dilemas éticos associados à início da vida}

Os artigos classificados nesta categoria foram aqueles que apresentaram questões éticas relacionadas a UTI neonatal (18), modo de morrer em UTI neonatal (13), limites do poder familiar sobre saúde dos seus filhos (14) e prematuridade extrema (15). Relacionado principalmente sobre as preferências dos pais dos bebes e das crianças hospitalizadas, além de discutir sobre os mecanismos envolvidos com a morte. Nenhum dos artigos avaliados referencia consulta aos comitês de bioética hospitalar e/ou metodologias de apoio a decisão.

\section{Dilemas éticos associadosao final da vida}

Um autor aborda o dilema dos envolvidos com os cuidados de pacientes de fim de vida (14) envolvendo questões como dignidade e qualidade. A importância do respeito a autonomia e ponderação de quem toma as decisões. Em outro artigo (17) discute-se sobre atitudes discriminatórias na admissão de idosos em UTI(s) e fragilidade da sustentação desta conduta baseado unicamente em dados da idade cronológica. A parte da argumentação foi realizada utilizando o referencial teórico do principialismo.

Wittmann-Vieira avaliou a qualidade de vida de 89 pacientes adultos oncológicos em cuidados paliativos. $O$ seu interesse estava relacionado com aspectos relativos à qualidade e autonomia dessas pessoas. Os seus dados demonstraram 
capacidade preservada para tomar decisões em seu melhor interesse, num cenário em que a vida não é mais o foco de atenção primária, o viver mantem-se como questão fundamental e portanto, a reflexão bioética do processo de tomada de decisão está sempre presente (23).

Apenas o artigo (14) propõe metodologias de apoio a decisão utilizando o fluxograma de Schramm, segundo o qual em face da conflituosidade intrínseca das relações humanas, a Bioética de proteção é uma ferramenta capaz de resolver os conflitos éticos, já que busca construir convergências por meios de princípios capazes de responder aos conflitos éticos, e, se possível respondê-los. Assim, propõe um fluxograma que deve ser observado para solução dos dilemas éticos: (a) ocupar-se de descrever os conflitos e compreendê-los da maneira mais racional e imparcial possível; (b) preocupar-se em resolvê-los, propondo as ferramentas que podem ser consideradas, por qualquer agente moral racional e razoável, mais adequadas para proscrever os comportamentos incorretos e prescrever aqueles considerados corretos; e (c) que, graças à correta articulação entre (a) e (b), fornecer os meios capazes de proteger suficientemente os envolvidos em tais conflitos, garantindo que cada projeto de vida seja compatível com os demais.

\section{Dilemas éticos associados à alocação de recursos}

Um artigo sobre seleção de pacientes em serviço de emergência (18), que orienta aos seus alunos (administração hospitalar) para análise de situações éticas hipotéticas sobre quem deve viver no cenário de emergência, evidencia diferenças significativas quanto a utilização de critérios válidos pelos participantes, chamando atenção a escolha pela condição econômica e social para quem deve ou não viver como critério.

O mesmo autor (18) retorna ao mesmo tipo de procedimento metodológico, diferindo apenas quanto a natureza dos entrevistados, agora com participação de pós-graduandos (mestrado e doutorado) em Saúde, mostrando clara tendência de escolhas orientadas pela ética utilitarista. Outra questão é abordada pelo artigo (21) quanto à limitação do esforço terapêutico na pessoa com lesão encefálica grave; indicando, com isso, que os elementos subjetivos permeiam a tomada de decisão dos envolvidos. Vale registrar a recomendação de fluxograma de Moritz para auxílio na tomada de decisão e recomendação de discussões multifacetas nos moldes de comitês de bioética hospitalar evitando, assim, decisões unilaterais, lineares e simplistas. 


\section{Dilemas éticos associados à realização de procedimentos terapêuticos}

No artigo (7), discute-se a hemotransfusão de pacientes Testemunha de Jeová, seu referencial religioso, opções alternativas de tratamento, situações envolvendo condições eletiva e de emergência, emitindo opiniões sobre legislação na época e declarando o conflito de dois princípios fundamentais: autonomia versus beneficência.

\section{Dilemas éticos associados à estratégia de tomada de decisão}

A utilização de sistemas computacionais de suporte a decisão em bioética clínica foi avaliada por SIQUEIRA-BATISTA (19) através de uma revisão integrativa da literatura. $O$ emprego de rede neurais artificiais - ou mesmo de outros métodos de aprendizado de máquina representam uma área em franca ascensão. No âmbito da bioética é ainda muito restrito com alguns exemplos pontuais na literatura de simulação de casos clínicos que permitem um apoio computacional efetivo para consecução do processo decisório dos médicos em cenário de final de vida.

Outra pesquisa, identifica situações na literatura que produzem angustia e desconforto na prática clínica e qual a abordagem que será utilizada para resolução dos dilemas entre os médicos envolvidos (15), o autor discorre sobre a importância da formação de comitês bioéticos que segundo o mesmo deverão ser multiprofissionais e multidisciplinares. Por fim, o autor identificou explicitamente diferentes metodologias de auxílio à tomada de decisão em bioética.

Pastura (17) discute que a bioética é frequentemente encarada como disciplina que respalda decisões em casos de conflito, indicando regras racionais e universais. De forma diversa, a ética das virtudes propõe que o indivíduo deva investir na construção do seu caráter e desta forma tomar decisões sábias e prudentes. Este conceito poderia ser extrapolado para os profissionais da área de saúde. $O$ autor realiza um ensaio reflexivo destacando as vantagens deste marco para tomada de decisões em âmbito médico.

Zoboli et al. (24) realizaram estudo que evidência a importância do uso de procedimentos sistemáticos e organizados para tomada de decisão, minimizando áreas de incertezas quanto aos conflitos de valores e deveres no âmbito clínico, visando alcançar decisões práticas, prudentes e responsáveis. Os autores descrevem os métodos casuísticos e a deliberação aplicados a um caso clínico à luz dos deveres éticos. 
Numa síntese das observações apresentadas nos tópicos anteriores, percebe-se a necessidade de ampliar-se a qualidade do cuidado em saúde por meio de instrumentos de apoio ao profissional de saúde, como os comitês de bioética hospitalar.

Há estudo (24) que propõe métodos, a casuísta e a deliberação, a serem utilizados pelos profissionais de saúde para tomada de decisão em casos clínicos. Recomendam esses métodos em razão do pluralismo moral que exige que os profissionais de saúde desenvolvam hábitos, habilidades e competências deliberativas para aprimorar a qualidade da atenção à saúde. Os métodos apresentados neste artigo iniciam-se com a compreensão do caso clínico. Tais procedimentos permitem decisões concretas, indicando um curso de ação realizável para solucionar o problema ético em foco. $\mathrm{Na}$ aplicação do método casuístico, por exemplo, analisa-se o caso clínico descoberto como problema ético em função dos tópicos: indicações médicas; preferências do doente; qualidade de vida e aspectos conjunturais. Em síntese, a deliberação de problemas éticos é a consideração dos valores e deveres intervenientes nos fatos concretos a fim de manejar a situação de conflito moral, de maneira razoável e prudente, por meio de discussões e decisões feitas no diálogo interpessoal. Visa soluções prudentes e não a decisão ideal, certa ou que maximiza resultados.

Outro estudo (23) assinala que a discussão bioética está presente na tomada de decisão, mas acaba o médico sobrepondo o conhecimento técnico à autonomia da vontade do paciente (paternalismo médico) por estar convicto de que faz o melhor para o paciente, ou seja, a ocorrência do paternalismo se dá quando o princípio da beneficência é praticado com desrespeito à autonomia do paciente. $O$ conflito na aplicação dos princípios do respeito à autonomia do paciente e o da beneficência para solução de dilemas éticos também foi verificado em outro artigo (22).

Por outro lado, há estudo (15) que apresenta uma crítica ao principialismo de Beauchamp e Childress, teoria adotada na maioria dos casos de tomada de decisão clínica. Os autores propõem que a discussão e reflexão sobre dilemas éticos sejam realizadas adotando-se outros fluxos da bioética, propondo o desenvolvimento de novos modelos teóricos para solução de conflitos éticos, bem como recomendam novas estratégias para apoiar a tomada de decisão. Estas novas estratégias devem considerar, entre eles 1) consulta a comissões (bio) éticas; 2) aplicação de uma abordagem computacional; e 3) procedimentos que permitem a abordagem pragmática da relação entre meios, fins e as pessoas envolvidas, possibilitando a tomada de decisões processo para incluir valores e sistemas preferenciais que são razoáveis e prudentes. 
Dessa forma, os artigos, em sua maioria, demonstram a necessidade de adoção de métodos e/ou procedimentos de apoio ao profissional de saúde na solução dos dilemas éticos. Sinalizam que os comités de bioético desempenham relevante papel na solução de casos médicos que exigem uma avaliação ética de maneira mais ampla; destacando, ainda, que o seu objetivo não deve ser apenas para discutir essas situações mais complexas advindas dos avanços tecnológicos na medicina, deve ser também meio de discussão de situações do dia a dia da assistência à saúde.

Esses comités são apresentados como instrumentos valiosos para o aperfeiçoamento dos profissionais dos hospitais e permitem um acolhimento de qualidade ao paciente e seus familiares, mas apesar da sua importância no auxílio a tomada de decisão pelo profissional de saúde, não fazem parte da cultura dos hospitais brasileiros.

\section{Considerações finais}

Os comitês bioéticos podem ser definidos como um corpo interdisciplinar de pessoas que têm como objetivo investigar e assessorar em questões éticas hospitalares. Representam um avanço ao auxiliar equipes assistenciais na tomada de decisões difíceis. A sua origem remonta aos EUA, com destaque aos casos judiciais que mobilizaram a opinião pública e do descompasso entre avanços na saúde e os critérios de escolha dos beneficiários, daí em diante, expandiu-se por todo o mundo.

Segundo dados dos EUA, em 1983 menos de $1 \%$ dos hospitais possuíam comitês de bioética em seus quadros. No início dos anos noventa, sua formalização tornou-se real com o apoio da Associação Médica Americana. A sua implantação é uma peça considerada importante para resolução de conflitos bioéticos pelos organismos acreditadores internacionais como Acreditação Canadense (QMENTUM) e Joint Comission on Acreditation of Healthcare e critério obrigatório de qualidade e credenciamento de hospitais nestes países. Atualmente mais de $90 \%$ dos hospitais americanos contam com comitês de bioética hospitalar.

Em sintonia com os estudos apresentados nesta revisão sistemática, percebe-se que as evidências da sua presença, e, por consequente, o encaminhamento dos conflitos éticos à comitês, parece ser ainda muito pouco expressivo no nosso país.

Isto tem consequências. Muitas decisões bioéticas acabam sendo tomadas por autoridades hospitalares e/ou profissionais do campo jurídico, não instrumentalizados com o saber bioético, apresentando respostas que procuram, na verdade, resguardar 
juridicamente, os entes jurídicos públicos e privados de eventuais demandas judiciais, ao invés de trazer soluções éticas que possam confortar o paciente e seus familiares.

O resultado da revisão literária demonstra que ainda estamos distantes de um modelo de saúde em que o atendimento ao melhor interesse do paciente seja o eixo central das tomadas de decisões em questões saúde.

\section{Referências}

1. Baumann-Hölzle, R., Maffezzoni, M., \& Bucher, H.U. (2005). A framework for ethical decision making in neonatal intensive care.Acta Peadiatrica, Interantional Journal of Peadiatrics, 94(12), 1777-1783.

2. Beauchamp, Tom and Childress, James. 2001. Principles of Biomedical Ethics, 5th edition. Oxford University Press.

3. Bethlem AS. Modelos de processo decisório. Rev Adm. 1987; 22(3):27-39.

4. Bezerra, Andrezza de Lemos et al. Ética na decisão terapêutica em condições de prematuridade extrema. Rev. Bioét. [Online]. 2014, vol.22, n.3, pp.569-574.

5. Carvalho, P. R. A.; Rocha, T. S.; Santo, A. E. E Lago, P. Modos de morrer na UTI pediátrica de um hospital terciário. Rev. Assoc. Med. Bras. [online]. 2001, vol.47, n.4, pp.325-331.

6. De Boer, J.C., van Blijderveen, G., van Dijk, G. Duivenvoorden, H.J., \& Williams, M. (2012). Implementing structured, multi-professional medical ethical decision-making in a neonatal intensive care unit.Journal of Medical Ethics, 38(10), 596601.

7. Franca, Inacia Sátiro Xavier de; Baptista, Rosilene Santos e Brito, Virgínia Rosana de Sousa. Dilemas éticos na hemotransfusão em Testemunhas de Jeová: uma análise jurídico-bioética. Acta paul. enferm. [Online]. 2008, vol.21, n.3, pp.498-503.

8. Fortes, Paulo Antonio De Carvalho. Selecionar quem deve viver: um estudo bioético sobre critérios sociais para microalocação de recursos em emergências médicas. Revista da Associação Médica Brasileira, Jun 2002, Volume 48 № 2 Páginas $129-134$.

9. Fortes, Paulo Antônio de Carvalho e Pereira, Patricia Cristina Andrade. Priorização de pacientes em emergências médicas: uma análise ética. Rev. Assoc. Med. Bras. [online]. 2012, vol.58, n.3, pp.335-340.

10. Freitas, Edna Estelita Costa e Schramm, Fermin Roland. Argumentos morais sobre inclusão/exclusão de idosos na atenção à saúde. Rev. Bioét. [Online]. 2013, vol.21, n.2, pp.318-327. 
11. Gomes, Henrique de Alencar et al. Limitação de esforço terapêutico na pessoa com lesão encefálica grave. Rev. Bioét. [Online]. 2014, vol.22, n.2, pp.282-290.

12. Kawamura K, Vicensi MC, Nodari RJ, Schlemper Junior BR, Bonamigoel. Comitê hospitalar de bioética: êxitos e dificuldades. Rev. bioét. (Impr.). 2012;20(1):140-9.

13. Kipper, Délio José. Limites do poder familiar nas decisões sobre a saúde de seus filhos - diretrizes. Rev. Bioét. [Online]. 2015, vol.23, n.1, pp.40-50.

14. Lima, Maria de Lourdes Feitosa; Rego, Sérgio Tavares de Almeida e Siqueira-BATISTA, Rodrigo.Decision-making process in end-of-life care. Rev. Bioét.[Online]. 2015, vol.23, n.1, pp.31-39.

15. Motta, Luís Claudio de Souza; Oliveira, Lucas Nicolau de; Silva, Eugenio e Siqueira-Batista, Rodrigo.Decision-making in clinical (bio) ethics: contemporary approaches. Rev. Bioét.[Online]. 2016, vol.24, n.2, pp.304-314.

16. Neves MPC, Oswald W. Bioética simples. Lisboa: Verbo; 2007.

17. Pastura, Patrícia Souza Valle Cardoso e Land, Marcelo Gerardin Poirot. A perspectiva da ética das virtudes para o processo de tomada de decisão médica. Rev. Bioét. [Online]. 2016, vol.24, n.2, pp.243-249.

18. Ribeiro, Carlos Dimas Martins e Rego, Sergio. Bioética clínica: contribuições para a tomada de decisões em unidades de terapia intensiva neonatais. Ciênc. saúde coletiva [online]. 2008, vol.13, suppl.2, pp.2239-2246

19. Siqueira-Batista, Rodrigo et al. Modelos de tomada de decisão em bioética clínica: apontamentos para a abordagem computacional. Rev. Bioét. [Online]. 2014, vol.22, n.3, pp.456-461.

20. sorokin, Patricia; Mariel and Outomuro. Comités de ética assistencial: de los grandes dilemas a los nuevos desafíos.Rev. Bioét. [Online]. 2016, vol.24, n.1, pp.91-97.

21. Tealdi JC, Mainetti JA. Hospital ethics committee.In: Connor SS, Fuenzalida-Puelma HL. Bioethics: issues and perspectives. Washington D.C.: Pan American Health Organization, 1990.p.52-8. Scientific publications, n. 527

22. Thomas L, Galla C. Building a culture of safety through team training and engagement. BMJ Qual Saf. 2013;22(5):425-34.

23. Wittmann-Vieira, Rosmari e Goldim, José Roberto. Bioética e cuidados paliativos: tomada de decisões e qualidade de vida. Acta paul. enferm. [Online]. 2012, vol.25, n.3, pp.334-339.

24. Zoboli, Elma. Tomada de decisão em bioética clínica: casuística e deliberação moral. Rev. Bioét. [Online]. 2013, vol.21, n.3, pp.389-396.

Recebido: 27/05/2019. Aprovado: 29/07/2019. 